\title{
DNA methylation differs extensively between strains of the same geographical origin and changes with age in Daphnia magna
}

\author{
Jack Hearn ${ }^{1 *}\left(\mathbb{D}\right.$, Fiona Plenderleith ${ }^{2,3}$ and Tom J. Little
}

\begin{abstract}
Background: Patterns of methylation influence lifespan, but methylation and lifespan may also depend on diet, or differ between genotypes. Prior to this study, interactions between diet and genotype have not been explored together to determine their influence on methylation. The invertebrate Daphnia magna is an excellent choice for testing the epigenetic response to the environment: parthenogenetic offspring are identical to their siblings (making for powerful genetic comparisons), they are relatively short lived and have well-characterised inter-strain life-history trait differences. We performed a survival analysis in response to caloric restriction and then undertook a 47-replicate experiment testing the DNA methylation response to ageing and caloric restriction of two strains of D. magna.
\end{abstract}

Results: Methylated cytosines (CpGs) were most prevalent in exons two to five of gene bodies. One strain exhibited a significantly increased lifespan in response to caloric restriction, but there was no effect of food-level $\mathrm{CpG}$ methylation status. Inter-strain differences dominated the methylation experiment with over 15,000 differently methylated CpGs. One gene, Me31 b, was hypermethylated extensively in one strain and is a key regulator of embryonic expression. Sixty-one CpGs were differentially methylated between young and old individuals, including multiple CpGs within the histone $\mathrm{H} 3$ gene, which were hypermethylated in old individuals. Across all age-related CpGs, we identified a set that are highly correlated with chronological age.

Conclusions: Methylated cytosines are concentrated in early exons of gene sequences indicative of a directed, nonrandom, process despite the low overall DNA methylation percentage in this species. We identify no effect of caloric restriction on DNA methylation, contrary to our previous results, and established impacts of caloric restriction on phenotype and gene expression. We propose our approach here is more robust in invertebrates given genome-wide CpG distributions. For both strain and ageing, a single gene emerges as differentially methylated that for each factor could have widespread phenotypic effects. Our data showed the potential for an epigenetic clock at a subset of age positions, which is exciting but requires confirmation.

Keywords: DNA methylation, Life-history traits, Variation, Daphnia, Ageing, Epigenetic

*Correspondence: Jack.Hearn@lstmed.ac.uk

${ }^{1}$ Department of Vector Biology, Liverpool School of Tropical Medicine, Liverpool, UK

Full list of author information is available at the end of the article

\section{Background}

Many progressive diseases of ageing in humans are associated with epigenetic modifications, the structural modifications on DNA that influence gene regulation. The relationship between epigenetic control and lifespan in humans (and many model systems) is complicated by variation in ageing rates between diet and genotype. The clonally reproducing invertebrate Daphnia magna is a

c) The Author(s) 2021. This article is licensed under a Creative Commons Attribution 4.0 International License, which permits use, sharing, adaptation, distribution and reproduction in any medium or format, as long as you give appropriate credit to the original author(s) and the source, provide a link to the Creative Commons licence, and indicate if changes were made. The images or other third party material in this article are included in the article's Creative Commons licence, unless indicated otherwise in a credit line to the material. If material is not included in the article's Creative Commons licence and your intended use is not permitted by statutory regulation or exceeds the permitted use, you will need to obtain permission directly from the copyright holder. To view a copy of this licence, visit http://creativeco mmons.org/licenses/by/4.0/. The Creative Commons Public Domain Dedication waiver (http://creativecommons.org/publicdomain/ zero/1.0/) applies to the data made available in this article, unless otherwise stated in a credit line to the data. 
tractable model for disentangling these effects. Daphnia have lifespans that can be studied within a reasonable time frame ( $\sim 50$ to 100 days in the laboratory), different strains are known to show natural variation in longevity, and in response to caloric restriction [1]. We can separate genetic from environmental effects in this species through genetically identical sister-clone replicates. Daphnia magna therefore enables experiments that assess the response of DNA methylation to ageing and known lifespan-extending treatments, such as caloric restriction (CR) $[2,3]$. Prior to this study, these interactions have not been explored together to determine their influence on DNA methylation.

\section{Arthropod DNA methylation and inter-genotype diversity}

DNA methylation is enriched in exon sequences of various arthropods, including members of holometabolous insect orders Lepidoptera and Hymenoptera [4-7] and species of the Crustacea genus Daphnia [8, 9]. Exonenriched DNA methylation has been shown to occur in genes expressed across multiple tissues $[6,7,10,11]$. This is not the only pattern of DNA methylation observed in arthropods, however. Hemimetabolous insects, such as cockroaches and termites, and the Crustacean Crassostrea gigas have higher genome-wide levels of DNA methylation that do not show exon bias to the extent of holometabolous insects and Daphnia [7, 12, 13]. The effect of genetic diversity on patterns of DNA variation has not been assessed in previous arthropod methylation studies. Knowledge of this has come from mammalian studies: in mice, for example, thousands of methylated cytosines (CpGs) have been found to differ between strains $[14,15]$, which was associated with proximity to DNA polymorphisms $[14,16]$. Inter-individual polymorphism in DNA methylation has also been observed in humans $[17,18]$.

\section{DNA methylation and ageing}

Mammals have high levels of CpG methylation genome wide $(\sim 70 \%)$, and degradation of methylation mechanisms are associated with cancers, diabetes and other conditions [19]. Furthermore, it is well established that CpG methylation status can be used to accurately predict chronological age in mammals [20,21]. This was first discovered in humans, but markers have been developed for a variety of mammals and a bird species [22-26]. Such 'epigenetic clocks' can be used to predict acceleration in the ageing process due to smoking or other factors with negative health effects. Dissecting the interactions between ageing and methylation regulation in Arthropods has lagged by comparison to mammals, although pharmacological inhibition (by RG108) of DNA methyltransferase activity was shown to increase lifespan in honeybees [27]. DNA methylation has been correlated to phenotypes in several insect orders: it shows sex-specific patterns in the Hemipteran aphids and mealybugs [28, 29], nurse and forage caste-specificity in honeybees [30], and is essential to larval development in the almost negligibly methylated mosquito species Anopheles albimanus [31]. Despite these correlations, causative links between phenotype and DNA methylation have yet to be established in arthropods [7].

\section{Caloric restriction and DNA methylation}

Caloric restriction $(\mathrm{CR})$ is the reduction of dietary intake without malnutrition or loss of micronutrients. It can increase lifespan in many animals, including $D$. magna [32-38]. CR acts through several molecular mechanisms which are possibly master regulated by the mechanistic target of rapamycin (mTOR) pathway [39]. CR in mice causes genome-wide remodelling DNA methylation that delays age-related changes [40]. However, in honeybees lifespan increases were observed on inhibition of DNA methyltransferase, but this was shown to occur in a CRindependent manner [27].

\section{An epigenomic model invertebrate-Daphnia magna}

Under favourable ecological conditions [41], Daphnia species reproduce clonally. Over time this results in high within-population genetic variation, as multiple non-recombining lineages are maintained [42-45]. Life-history experiments have exploited this variation to explore how distinct genotypes (or strains) of a variety of Daphnia species respond to environmental stressors $[1,46-52]$. D. magna has more recently emerged as an epigenetic model organism. A variety of life-history and ecotoxicological stimuli have been shown to modify methylated cytosines (CpGs), histones and small RNA expression [1, 3, 9, 53-60]. In this role, Daphnia species have an advantage over the model invertebrate Drosophila melanogaster. D. melanogaster has negligible levels of adult DNA methylation and lacks the essential DNA methyltransferase genes (DNMT1 and DNMT3A/B), although this is subject to continuing debate (discussed in [61]). D. magna encodes a full complement of DNMTs and gene-body DNA methylation has been shown to correlate with increased gene expression in male versus female $D$. pulex [9]. This is despite low levels of overall cytosine $(\mathrm{CpG})$ methylation of $0.25-0.8 \%$ estimated in D. magna $[8,53-59]$, which is sparsely distributed across Daphnia genomes $[8,9,53,55,56,58,59]$. Hence, DNA methylation is likely a directed and non-random process in Daphnia that has a role in the regulation of gene expression. 


\section{A multi-factor experimental design}

We have previously shown that $\mathrm{CR}$ causes differential methylation, gene and miRNA expression in D. magna [1, 3 , 59]. However, we found no correlation between differentially methylated regions and gene expression resulting from CR [1], which necessitated confirmation of any link between CR and CpG methylation in this study. This lack of correlation also informed our choice to take a different approach to identifying strain, age and CR changes in CpG methylation here. Firstly, smoothing-based approaches to identify changes in methylation status (as we applied before [59]) combine methylated cytosines in close proximity, were developed with the high levels of DNA methylation in vertebrates as a basis. Secondly, our experimental design is complex incorporating two strains, abundant versus calorically restricted food levels and young versus old individuals (detailed in Fig. 1a). We therefore applied a generalised-linear modelling approach to identify individually differentially methylated CpGs [62]. The advantage of this approach over alternatives (discussed in [54]) is that no assumptions were made about the underlying distribution of DNA methylation, and we were able to assess all experimental factors in one model.

We first conducted a survival analysis of CR on our two strains, which showed that one strain has increased longevity in response to $C R$ but the other does not. Then we tested if genome-wide methylation status varies by Age, $\mathrm{CR}$ and Strain. Strain is by far the strongest factor in the experiment accounting for the majority of significant

a

C32 and KA53
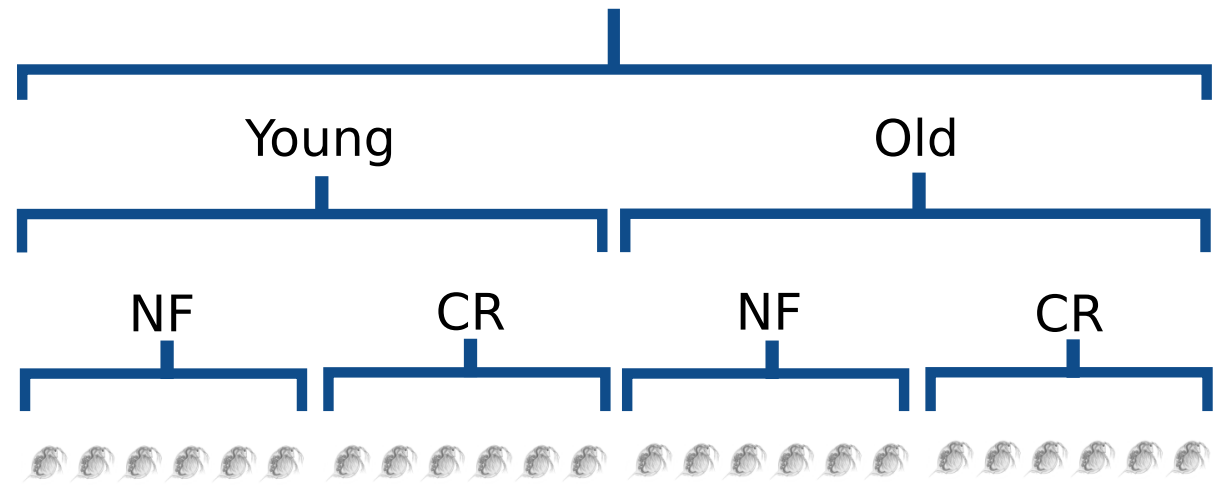

b

C32
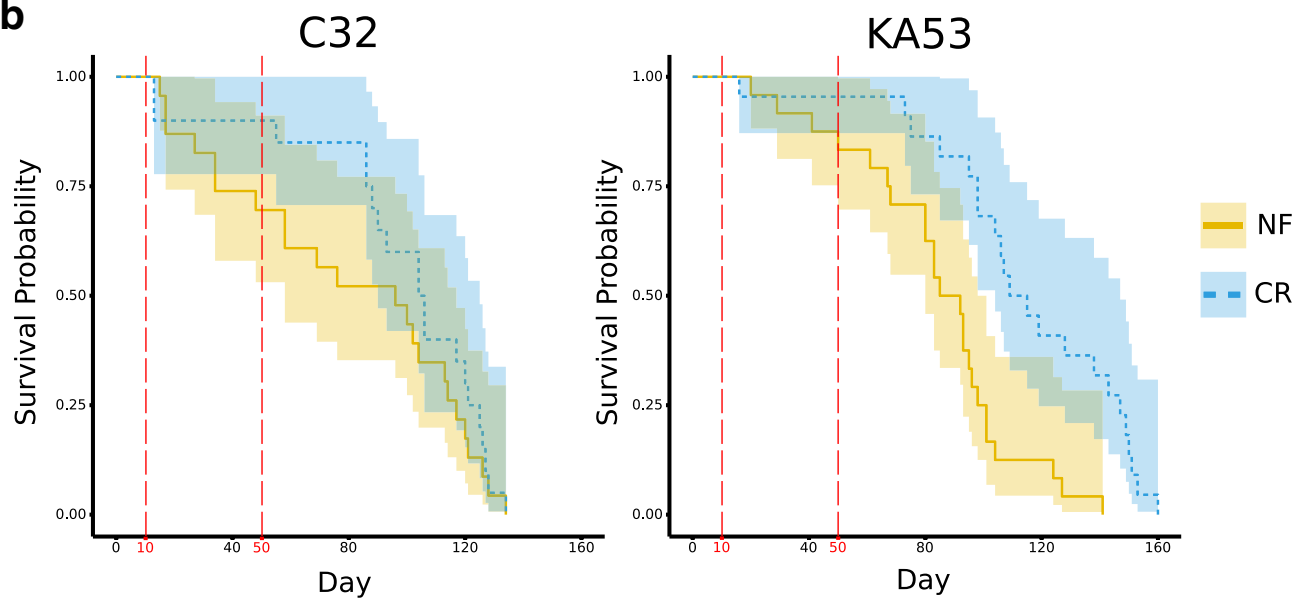

Fig. 1 Ageing and response to caloric restriction in strains C32 and KA53. a Experimental design for each strain, half of replicates were sampled at 10 days and half at 50 days and half of replicates were submitted to normal food (NF) and half to caloric restriction (CR). This resulted in eight combinations of factors (two strains $x$ two ages $x$ two food levels), each of which consisted of six replicates. b Survival curves for C32 and KA53 in response to caloric restriction and normal food levels. $\mathrm{NF}=$ normal food; $\mathrm{CR}=$ caloric restriction $\mathrm{X}$-axis $=$ time in days; orange line $=$ survival probability for normal food; orange shading $=95 \%$ confidence interval of the normal food survival probability; blue dashed line $=95 \%$ confidence interval of the caloric restriction survival probability; $C R=$ caloric restriction; red dashed vertical lines indicate sampling points for young replicates at 10 days, and old replicates at 50 days 
differentially methylated CpGs. Age was significant at much fewer CpGs, but these positions predict epigenetic ages that are highly correlated with chronological age, hinting at the potential for defining an epigenetic clock in Daphnia. In contrast to our previous research, CR had no consistent effect on methylation.

\section{Results}

\section{Strain lifespan and response to $C R$}

The two strains, known to us as strain C32 and KA53, respond differently to caloric restriction (survival curves: Fig. 1b). Median survival time in strain KA53 changed from 89 days (95\% confidence interval: $80-98$ days) to 112 (95\% CI 104-147) with a likelihood ratio test $p$-value of $2 \times 10^{-4}$ in response to CR. For C32, median survival increased from 96 days ( $95 \%$ CI 58-117) to 105 days (95\% CI 90-125), but this difference was not significant ( $p$-value 0.4 , likelihood ratio test).

\section{WGBS metrics}

MultiQC collated mapping, conversion rates and other metrics per replicate are given in Additional files 1 and 2 for the two filtering methods: (1) to control for incomplete bisulphite conversion, reads with three or more consecutive unconverted $\mathrm{CH}$ cytosines were removed ('3xuCH') as recommended by Ref. [63], and (2) all reads containing $\mathrm{CH}$ methylated cytosines were removed ( 'No-CH') in line with our previous DNA methylation study Daphnia [59]. Mapping rates were highly correlated between the reference genome [64] and D. magna version 2.4 genome assembly [65], with a Spearman's rho of 0.997 ( $p$-value $2.2 \times 10^{-16}$ ). The low mapping rates of some replicates (lowest 29.8\% reads aligned; highest 66\%) do not result from a different alignment reference from our previous study [59]. Average percentage CpG methylation was $1.26 \%$ and $1.33 \%$ for the 'No- $\mathrm{CH}$ ' and ' $3 \mathrm{xuCH}$ ' datasets, respectively, (column: ' $\% \mathrm{mCpG}$ ', Additional files 1 and 2), while cytosine coverages varied from 8 to 28 reads for both filtered datasets (column: 'C Coverage' Additional files 1 and 2).

\section{Replicates separated clearly by Strain}

During Initial data exploration, we discovered that one sample of strain C32 clustered with KA53 (Sample 5, Additional file 3: Figure S1), which we believe to have been a contamination or mislabelling error. This replicate, a strain C32-age Old-food level Normal Food, was removed from the analysis, and all results described were based on analysis of the remaining 47 replicates.

PCA and hierarchical clustering of the 10,000 most variable methylated $\mathrm{CpG}$ sites both show separation of replicates by Strain (Fig. 2a, b). The first PCA component explained $75.4 \%$ of the variance and the second $1.2 \%$, and the hierarchical clustering separated replicates by strain with $100 \%$ support from approximately unbiased p-value and bootstrap probabilities. CpGs with greater than 5\% methylation were concentrated in coding sequences, with 2.2 CpGs per kilobase versus untranslated regions (1.0 CpGs per $\mathrm{kb})$, introns (0.8 per $\mathrm{kb}$ ) and transcription start $(0.8$ per $\mathrm{kb})$ and end sites $(0.8$ per kb) (Fig. 2c, Tabular results: Additional file 4: Table S1), and there is no difference in these distributions between strains (Additional file 5: Figure S2). When broken down by exon, CpGs occurred more frequently in exons two to five, with the highest frequency in exon two at 3.6 CpGs per kb (Fig. 2 part D, tabular results: Additional file 6: Table S2), and the distribution across exons was not different between strains (Additional file 5: Figure S2).

\section{Strain explains most significant $\mathrm{CpGs}$}

A total of 4,141,957 and 4,095,469 CpGs were input to DSS for the ' $3 \mathrm{x} u \mathrm{CH}$ ' and 'No-CH' datasets, respectively. For the ' $3 \mathrm{x} u \mathrm{CH}$ ' dataset, 15,139 significant CpGs were associated with Strain, and $61 \mathrm{CpGs}$ were significant for Age. There were fewer significant sites for the 'No$\mathrm{CH}^{\prime}$ dataset for both factors (Table 1, Additional file 7: Table S3 for significant CpGs per filtering level and factor). No sites were significant for food level, nor were any interaction terms in the ' $3 \mathrm{x} u \mathrm{CH}$, ' $\mathrm{No}-\mathrm{CH}$ ' and unfiltered datasets. Strikingly, $87 \%(13,229 / 15,139$ CpGs) of differentially methylated $\mathrm{CpGs}$ for Strain in this ' $3 \mathrm{x} u \mathrm{CH}$ ' dataset occur in gene bodies (exons + introns) versus inter-genic sequence. To place this number into context, $49 \%$ of the genome assembly $(60,352,426$ bp out of $122,952,669)$ is covered by gene body sequence. This was also true for the 'No-CH' dataset at $88 \%(12,282 / 14,011$ CpGs) (list of significant CpGs: Additional file 8: Table S4). Compared to the unfiltered dataset, the results for Strain were very similar to the ' $3 \mathrm{x} u \mathrm{CH}$ ' dataset at 15,139 versus 15,150, but not for Age: 6,978 CpGs were significant in the unfiltered versus the 61 for the ' $3 \mathrm{x} u \mathrm{CH}^{\prime}$ dataset (Additional file 7: Table S3).

Overlap between the two filtering approaches is high for Strain significant CpGs with 13,834 CpGs in common (Euler diagram, Additional file 9: Figure S3). For Age, all four significant 'No-CH' CpG sites were present in the ' $3 \mathrm{x} u \mathrm{CH}$ ' significant $\mathrm{CpGs}$. Despite the strong differentiation between strains, there are very few genes that contain CpGs exclusive to either C32 or KA53, and in most cases this is only 1-5 CpG sites. An exception to this is gene LOC116934226, encoding an ATP-dependent RNA helicase me31b-like protein, which had 18 sites hypermethylated in strain KA53 versus C32; 12 in exon three, four in exon four and two in exon five: Of these significant CpGs, only one from exon, four and two from exon, and five occurred within protein coding sequence 


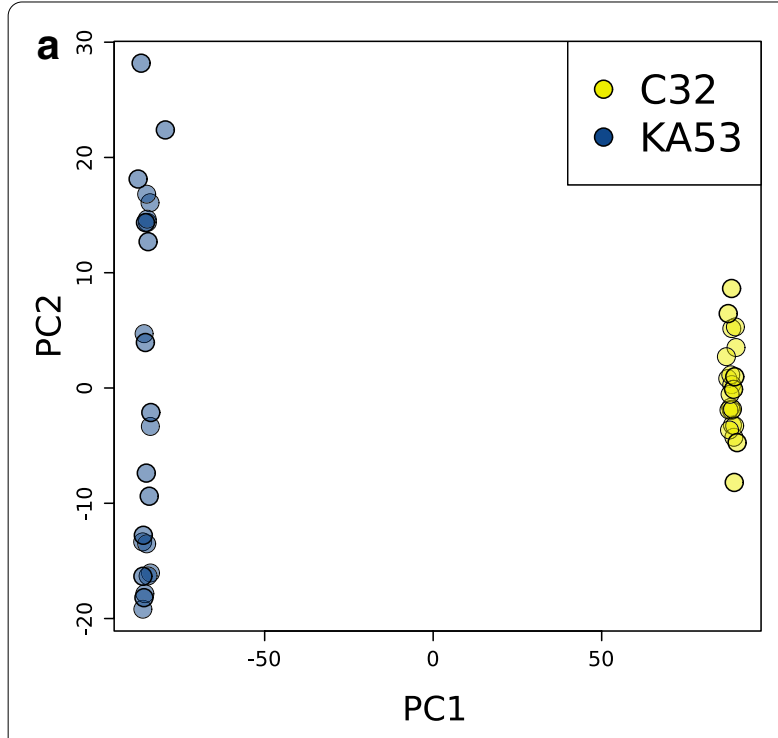

C
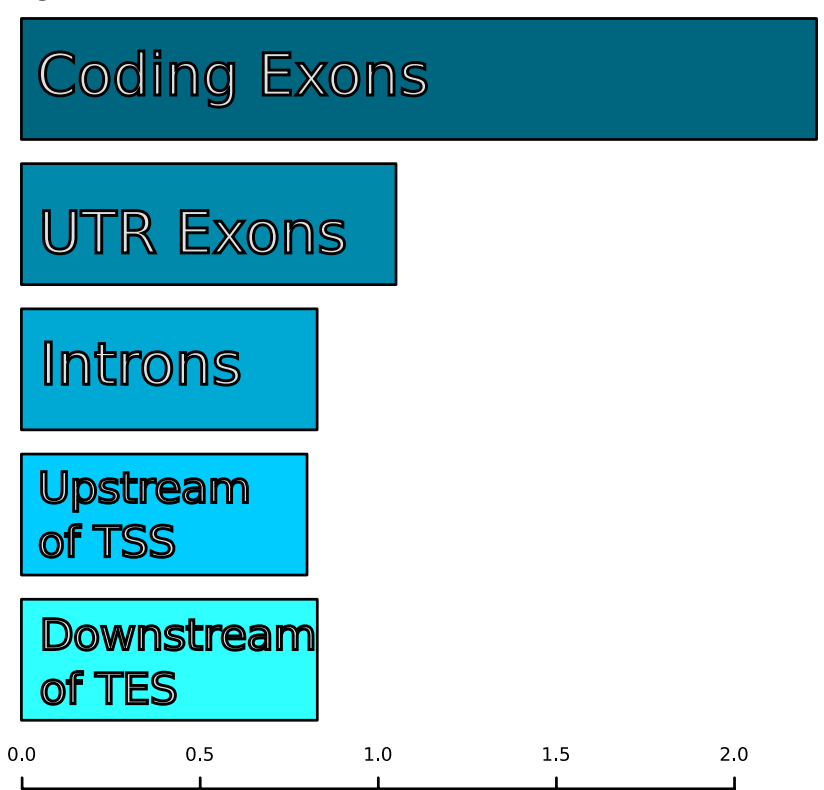

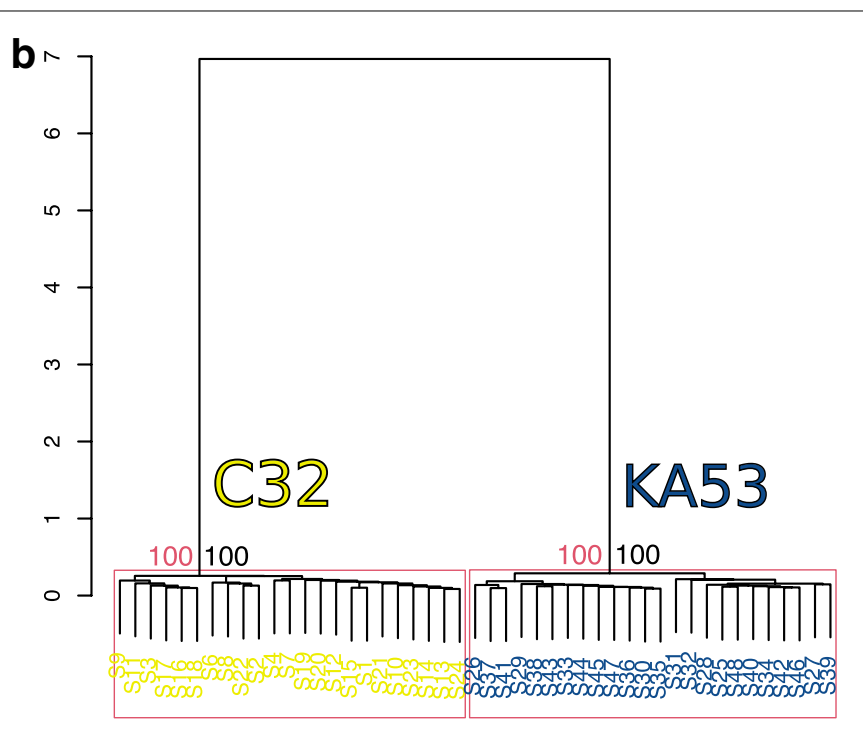

d
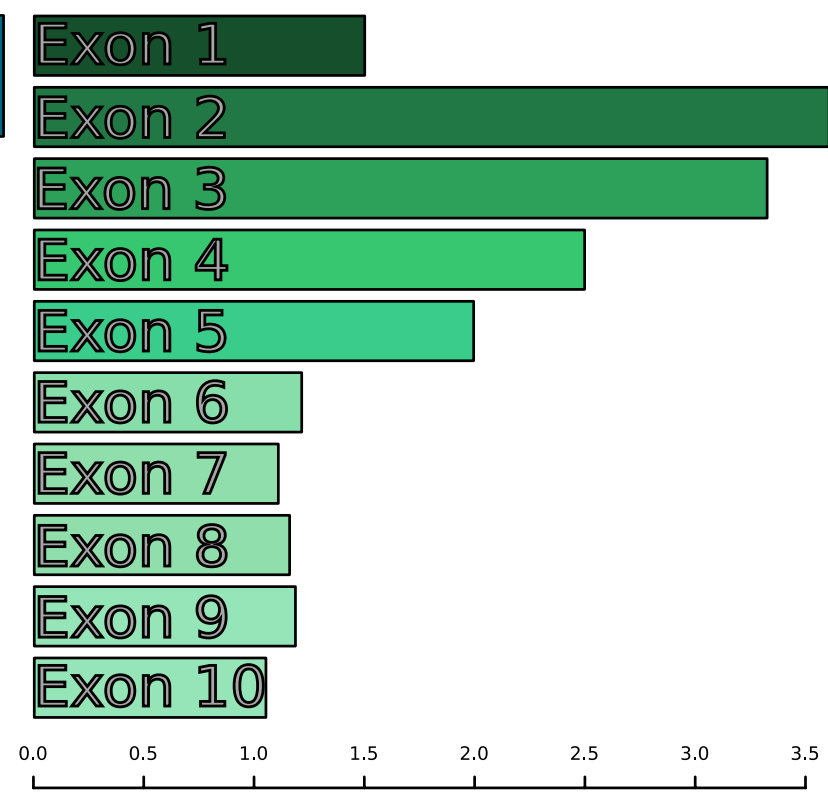

CpG per kilobase

Fig. 2 Methylation status correlates with Strain. a PCA plot of replicates for principal components one and two for the top 10,000 most variably methylated CpGs by beta value; C32=yellow, KA53= blue. $\mathbf{b}$ Hierarchical clustering of replicates with colouring as for $\mathbf{a}$ ), replicates split by strain with $100 \%$ approximately unbiased $p$-value (red) and bootstrap probabilities (black). c CpGs with greater than 5\% methylation combined across replicates are enriched in the coding regions of exons versus the rest of gene bodies. $\mathbf{d} \mathrm{CpGs}$ with greater than $5 \%$ methylation combined across replicates are concentrated in exons two to five in line with previous studies investigating DNA methylation in D. magna [8]

(Additional file 10: Table S5) with the rest present in the $5^{\prime}$ UTR.

BS-SNPer predicted 317,986 and 424,261 single-nucleotide polymorphisms (SNPs) from the WGBS data in strains C32 and KA53, respectively; of these 30,817 SNPs were exclusive to C32 and 137,092 to KA53. Compared against the reference assembly of South Korean origin,
287,169 SNPs were shared by both United Kingdom collected strains, a further 100,085 of which were fixed in both. Numbers of predicted SNPs were highly similar for the 'No-CH' BS-SNPer results (Additional file 7: Table S3).

There were few enriched GO terms relative to the number of genes containing exons with hypermethylated 
Table 1 Numbers of CpG sites hypermethylated in each strain relative to the other for Strain and Age factors in the ' $3 \times \mathrm{CH}^{\prime}$ ' and No-CH' filtered datasets

\begin{tabular}{|c|c|c|}
\hline \multicolumn{3}{|l|}{ Strain } \\
\hline Dataset & Hypermethylated in C32 & $\begin{array}{l}\text { Hypermethylated } \\
\text { in KA53 }\end{array}$ \\
\hline $3 \times \cup C H$ & 7787 & 7352 \\
\hline $\mathrm{No}-\mathrm{CH}$ & 7198 & 6813 \\
\hline \multicolumn{3}{|l|}{ Age } \\
\hline Dataset & Hypermethylated in Young & $\begin{array}{l}\text { Hypermethylated } \\
\text { in Old }\end{array}$ \\
\hline $3 \times u C H$ & 53 & 8 \\
\hline $\mathrm{No}-\mathrm{CH}$ & 3 & 1 \\
\hline
\end{tabular}

Table 2 Number of genes overlapped by significant CpG sites in exonic, coding sequence and intronic regions for all sites and genes with five or more hypermethylated CpGs

\begin{tabular}{llll}
\hline Strain & Exonic & CDS & Intronic \\
\hline C32 & 2625 & 1701 & 1495 \\
KA53 & 2469 & 1603 & 1364 \\
5 or more hypermethylated CpGs & & \\
C32 & 276 & 182 & 94 \\
KA53 & 292 & 185 & 100 \\
\hline
\end{tabular}

CpGs (Table 2). The category with most GO terms enriched was 'One or more hypermethylated CpGs' for biological process (BP) for C32 and KA53 at 21 terms for both (enriched GO terms for the eight comparisons made: Additional file 11: Table S6).

CpGs associated with age and a potential epigenetic clock Genes overlapping CpG sites significant for Age are given in Additional file 12: Table S7. For the 53 CpGs hypermethylated in Young Daphnia in the $3 \mathrm{xuCH}$ dataset, 35 overlap with gene bodies. All eight CpGs hypermethylated in Old replicates overlap a gene, five of which occur in the coding sequence of a single gene-histone H3 (LOC116920166). For the CpGs hypermethylated in Young individuals, one uncharacterised gene (LOC116933951) is overlapped by three significant CpGs, and nine further genes are overlapped by two significant CpGs, including prefoldin RPB5 interactor-like, homeobox protein Hox-B1a-like, innexin shaking-B-like, and an uncharacterised locus.

After filtering for CpGs with zero read coverage in any replicate, only one position was removed from the ' $3 \mathrm{x} u \mathrm{CH}$ ' Age significant CpGs (leaving 60 in total), which were analysed by PCA of beta values (the methylated proportion of cytosines at a single CpG, Fig. 3a). Young individuals of strain C32 separated clearly along PC1 which explains $66 \%$ of the variance in the data, while PC2 explained $8 \%$ of the variance. These 60 positions were input to the penalised lasso regression model, which revealed 12 sites that contributed significantly to the model (Additional file 13: Table S8). The relationship between epigenetic age and chronological age has a Spearman's rho of 0.87 ( $p$-value $3.9 \times 10^{-15}$ ) per replicate as predicted by these $12 \mathrm{CpGs}$ as shown in Fig. 3b. Strain KA53 had average epigenetic ages of 18.8 and 40.6 days at chronological ages of 10 and 50 days, respectively, whereas for C32 epigenetic ages increased from 11.6 to 49.0 days over the experiment. A GLM of the predicted ages per replicate extracted from the penalised regression indicated that Age explained $81 \%$ of the variance in the model, with clone explaining only $0.02 \%$ of the variance. However, the Strain by Age interaction explained a moderate amount of variation, 5.7\%. (results: Additional file 14: Table S9) in line with Fig. 3a in which Young replicates of C32 appeared distinct from the rest of the experiment. This was also true when only the twelve penalised regression selected CpGs are selected (Additional file 15: Figure S4, part A). The distinction between strains was greater when considering the four Age significant CpGs for the 'No-CH' filtered dataset, all of which contributed to the penalised regression (Additional file 13: Table S8). For that regression Strain explained $17 \%$ of the variance and was highly significant, as was Age at 34\%, and their interaction at 23\% (Additional file 13: Table S8 and Additional file 14: Table S9). The effect of strain can be seen in the correlation between chronological and epigenetic age, in which C32 replicates were all younger epigenetically than KA53 replicates at day 10 chronological age (Additional file 15: Figure S4, part B). 

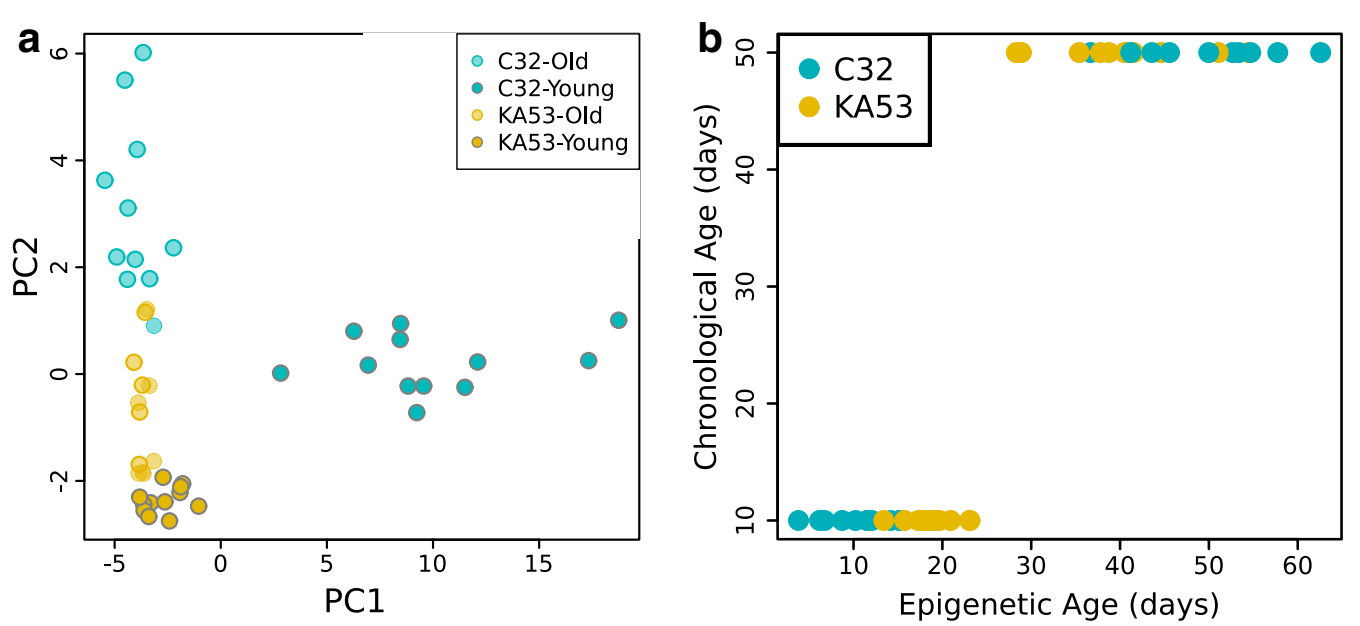

Fig. 3 Epigenetic age correlates with chronological age. a PCA analysis of beta values for 60 Age significant CpGs (one position was removed as it contained N/A values for certain replicates due to no read coverage) showing that C32 Young individuals cluster separately on PC1. b The relationship between predicted epigenetic age and chronological age for all replicates for the ' $3 \times \mathrm{xuCH}$ ' dataset, coloured by strain and calculated from the 12 CpGs that contributed most to the penalised lasso regression model

\section{Discussion}

\section{Methylated CpGs were enriched in exons}

Methylated CpGs were concentrated in gene bodies with a preference for exons 2 to 5 (Fig. 2d), adding to the evidence that methylation is a directed process in Daphnia $[8,9]$. We report an average CpG methylation of $1.2-3 \%$, which is higher than our previous estimate for strain C32 of 0.7\% [59]. This earlier estimate is more in line with those reported for other strains of $D$. magna at $0.25-0.85 \%$ [8, 9, 55-57]. The current higher estimate may result from incomplete bisulphite conversion during library preparation, as the percentage was elevated when filtering out all methylated $\mathrm{CHG} / \mathrm{CHH}$ sites (the 'No$\mathrm{CH}^{\prime}$ dataset) which occur negligibly in D. magna [56]. The CpG methylation rate of the mitochondrial genome has been used as a method of estimating non-conversion rate for D. magna before [58], as no mitochondrial DNA methylation occurs in the crustacean C. gigas [13]. Our data had a mitochondrial $\mathrm{CpG}$ average methylation rate of $0.8 \%$ for the ' $3 \mathrm{x} u \mathrm{CH}^{\prime}$ ' and $0.65 \%$ for the 'No- $\mathrm{CH}$ ' datasets, which when subtracted from the global CpG percentages (' $3 \mathrm{x} u \mathrm{CH}^{\mathrm{C}}$ ' dataset: $1.3-0.8 \%=0.5 \%$ ) were in the range of other estimates derived from $D$. magna. This lack of mitochondrial DNA methylation has not been confirmed in D. magna or any invertebrates beyond $C$. gigas [13], so the mitochondria method should be interpreted with caution (see [66] for mitochondrial CpG distributions in animals). We argue that by performing a well-replicated experiment in which there are 24 versus 23 replicates for each level of factor tested (Age/Strain/ Food), the effect of false-positive CpGs resulting from non-conversion of cytosines was minimised.

\section{CPG methylation did not respond to caloric restriction}

No CpGs were significant for CR at any filtering threshold despite this treatment having an effect on lifespan for KA53 (Fig. 1b) and other phenotypic effects on strain C32 $[1-3,59,67]$. This contrasts with our previous results for a CR-only $\mathrm{CpG}$ methylation experiment using C32 [59]. There was no association between median lifespan and CR for the survival analysis in this strain, but it does show previously established maternal effects in response to CR [67]. We believe this experiment to be a more robust test of CR than the previous research for two reasons: (1) our experiment includes more replicates of each food level ( 24 and 23 in total) and includes two strains; (2) the method used here to identify differentially methylated CpGs makes no assumptions about the underlying distribution of CpGs genome wide. Hearn et al. applied smoothing across linked CpGs to identify differentially methylated regions, an approach which was developed with much higher levels of methylation as a basis [68]. As a result, we recommend DSS-like methods that test significance of an individual CpG for arthropod studies until smoothing approaches that better model underlying $\mathrm{CpG}$ distributions in arthropods become available.

The lack of significant CpGs for food is at variance with previous miRNA and protein-coding gene expression experiments exploring responses to $\mathrm{CR}$ $[1,3]$. Greater than 6000 genes significantly differentially expressed in response to CR in C32 [1], but we found no overlap between gene expression response to $\mathrm{CR}$ and the previously identified differentially methylated regions in Ref. [1]. Given this very strong gene 
expression response, if changes in CpG methylation also formed part of the downstream response to CR in D. magna, we might expect to see differential methylation [69]. We conclude that the downstream responses to CR in D. magna do not cause changes in DNA methylation.

\section{Strain-dependent methylation differences were widespread}

We showed that two strains of D. magna that have $>15,000$ differently methylated CpGs for the ' $3 \mathrm{x} u \mathrm{CH}$ ' dataset. This is perhaps not surprising if genetic variability correlates with epigenomic differences as for vertebrates $[14,16]$. The maintenance of high-genetic diversity within natural Daphnia populations due to clonal lineages that result from extended periods asexual reproduction [42-44] could therefore amplify polymorphism at the sequence and methylation level versus sexually recombining species. We see support for this in the 30,817 and 137,092 SNPs exclusive to strains C32 and KA53, respectively. Both strains are distant from the South Korea-derived reference sequence as shown by the 287,169 SNPs shared between strains. These SNP numbers must be considered with care, however, as variant predictions derived from WGBS data are likely to be inflated by false positives from methylation polymorphisms [70, 71]. Strain-specific CpGs are concentrated in gene bodies across thousands of genes (Table 2), and the functional effects of such widespread differential methylation resulting from genetic variation are unknown.

One gene, LOC116934226, hypermethylated strongly at multiple CpGs in strain KA53 exclusively encode ATPdependent RNA helicase me31B-like protein. $M e 31 B$ is an RNA-binding protein that represses thousands of maternal mRNAs during Drosophila maternal-to-zygotic (MZT) transition [72, 73]. MZT is the process by which development comes under zygotic genome control over stored maternal genetic material in animals [74]. It is an essential gene for successful oogenesis and embryogenesis in Drosophila [74], with other mRNA-related decay functions that include antiviral activity in mosquitoes [75]. A methylation-dependent difference in embryonic expression levels of $m e 31 b$ could change developmental trajectories of embryos of each strain with phenotypic effect. The intergenerational stability of methylation at this locus will be a target of future work, by crossing these strains in future experiments will confirm whether CpG methylation in D. magna inheritance is linked to polymorphism.

GO term enrichment was less enlightening, possibly because strain can be considered a random effect within our experiment, albeit with only two levels. This means we do not know if the strains differ ecologically in their original environment, which makes it difficult to correlate general GO terms with phenotype in a biologically meaningful manner.

\section{Age resulted in DNA methylation changes}

For both filtering methods, there were significant CpGs for Age, although a ten-fold difference is observed between the ' $3 \mathrm{x} u \mathrm{CH}$ ' (61 CpGs) and 'No- $\mathrm{CH}^{\prime}$ (4 CpGs) datasets. PCA of the ' $3 \mathrm{x} u \mathrm{CH}$ ' Age significant $\mathrm{CpGs}$ reveals that Young replicates of strain C32 separate on PCA1 (Fig. 3a), suggesting that Strain can contribute to the Age effect. This is despite the lack of significant Strain by Age interaction CpGs from the DSS results, which may reflect a lack of power to detect such interactions under our experimental design and read coverage levels.

Five of the eight significant CpGs hypermethylated in Old individuals occurred in the histone $\mathrm{H} 3$ gene towards the $3^{\prime}$ end of coding sequence. This is the first observation of differential methylation along a histone gene in Daphnia as far as we are aware. Histone H3 is one of the five highly conserved histone genes that pack DNA into nucleosomes, and are of fundamental importance to organismal function [7]. It has several post-translational associations with heterochromatin (H3K9me3), activated transcription (H3K4me3/H3K36me3/H3K27ac), gene downregulation (H3K27me3) or a context-dependent effect (H3K4me1) [7]. Post-translational modifications of histones have been shown to differ between sexes in D. pulex [9]. Histone modifications are associated with behavioural transitions in ants and are important in head development of lepidoterans [7, 76-78]. From these observations, we predict that Increased methylation of histone H3 may increase this gene's expression with age with potentially organism-wide functional consequences. In support of this, increased DNA methylation has been shown to increase gene expression in D. magna and $D$. pulex $[8,9]$. However, this positive relationship between methylation and expression has not been shown to occur within an organism due to a treatment (where 'ageing' is the treatment). DNA methylation is not the only regulatory mechanism to differ with age in D. magna, as micro-RNA expression differs between young and old individuals and the eggs of young and old, respectively, in strain C32 [3]. Any interactions between miRNAs and constituents of chromatin, like histone H3, in arthropods have not yet been elucidated $[7,79]$ in contrast to the piwi-interacting RNAs [80], class of small RNAs.

Although more sites are significantly hypermethylated in young individuals, no gene or genes emerge with potentially large effects according to their function. Only uncharacterised gene 'LOC116933951' has more than two overlapping significant CpGs; it encodes an integrase domain and is present on a short contig of $5139 \mathrm{bp}$ 
length. This gene is likely a repetitive element that may have multiple genomic copies, which is supported by an average read coverage of 2426 for CpGs for data from all replicates combined (' $3 \mathrm{x} u \mathrm{CH}$ ' dataset) versus 661 for CpGs placed onto chromosomes. The lack of pattern in sites losing methylation with age could result from reduced efficiency of methylation maintenance protein DNMT1 [81], which is associated with lower expression with increasing age in humans [82]. Our sampling point of 50 days for Old replicates was constrained by a requirement for enough individuals to obtain sufficient DNA input for whole-genome bisulphite sequencing (WGBS). D. magna individuals can survive for longer [1, 83], and if DNMT becomes less efficient through lowered expression with age, we predict more hypomethylation at the same, and possibly more, CpG sites. Such studies are becoming feasible with newer methods exploring DNA methylation, especially when candidate CpG sites are targeted [26].

\section{Potential for an epigenetic clock in a model invertebrate}

In an exploratory analysis, we applied penalised lasso regression to the Age significant $\mathrm{CpGs}$ of the ' $3 \mathrm{xuCH}$ ' dataset which identified $12 \mathrm{CpGs}$ contributing significantly to the model specified. The interaction between Age and Strain was significant, but modest (6\%) compared to that of Age alone (81\%) at these CpGs Predicted or 'epigenetic ages' based on these twelve sites that were highly correlated with chronological age for replicates of both strains. (Fig. 3b). All 4 sites significant for the 'No$\mathrm{CH}$ ' dataset were kept by the model, but these positions were more strongly influenced by Strain, as young replicates of each clone had distinct epigenetic ages (Additional file 15: Figure S4, part B). This is despite the initial DSS analysis finding no CpGs with significant interactions between factors, possibly due to low power as millions of positions were included. Our results are the first, tentative, step in exploring clock-like mechanisms in an invertebrate. The twelve and 4 sites predicted here by the ' $3 \mathrm{x} u \mathrm{CH}$ ' and 'No-CH' datasets, respectively, require validation in multiple strains of $D$. magna for assessing whether a species-wide epigenetic clock is feasible. We plan to do this in future work, following the approach of Little et al. [26] for wild mice. The availability of such epigenetic age markers in tandem with the finely controlled life-history experiments commonly performed on $D$. magna has great potential for the field.

\section{Conclusions}

In summary, we found (1) no effect of caloric restriction on methylation, in contrast with prior work that used a different analysis approach; (2) more than 15,000 CpGs differentially methylated between strains sampled from the same habitat; and (3) that Age is associated with 61 differentially methylated CpGs, and some of these sites could prove useful for developing an epigenetic clock. Functional inferences are limited for the effect of Strain and Age on phenotype, but for each of these two factors, a single-gene emerges that could have a widespread phenotypic effect. For Strain, Me31b is a key embryonic repressor of maternal mRNAs, differential methylation of which leading to changed expression levels is hypothesised to put each strain on modified developmental trajectories. For Age, the hypermethylation of histone $\mathrm{H} 3$ may change expression levels between Young and Old individuals very broadly, as we have seen before for miRNAs. It is also a point of regulation between two epigenetic mechanisms, DNA methylation and chromatin, that has not been reported previously in Daphnia. Future work will focus for (2) on incorporating more strains from different locations to test whether epigenetic variation is more diverse within, or between, habitats. For (3) we now have a target set of CpG sites that we will test for clock-like behaviour in other strains of $D$. magna. The potential for an epigenetic clock in an easily manipulated, relatively short-lived, life-history trait model invertebrate for ageing research is very exciting.

\section{Methods}

\section{D. magna ageing and caloric restriction survival analysis}

Both strains originate from Kaimes pond near Leitholm, Scottish Borders, United Kingdom [84]. The experimental conditions specified below were the same as for the survival analysis presented in Ref. [1], and the results for strain C32 were first published in that study and reproduced here for comparison with KA53. Prior to the experiments, replicates of each strain were put through three generations of acclimation to harmonize environmental effects. During this period, each individual was maintained in a 60-ml glass jar filled with artificial pond medium [85] which was changed twice a week and after the birth of a clutch. Each individual was fed $\sim 6.25 \times 10^{6}$ Chlorella vulgaris cells daily and was maintained on a $12: 12 \mathrm{~h}$ Light:Dark cycle at $20{ }^{\circ} \mathrm{C}$. Offspring from the second clutch initiated each generation and the experimental generation. We define Caloric restriction as approximately $20 \%$ of normal food levels. From acclimated females of each strain, two offspring were taken, and one replicate was given normal food of $\sim 6.25 \times 10^{6}$ cells and the other given a calorically restricted $\sim 1.4 \times 10^{6}$ cells. Each food treatment and genotype combination were replicated 24 times and date of birth and date of death of all individuals in the experiment were recorded.

CR-induced differences in lifespan difference within each strain were tested by a Cox's proportional hazards model using the survival package ( $\mathrm{R}$ code, Additional 
file 16, strain longevity data: Additional file 17) [86]. Between strain differences were not tested as the experiments were run separately, but under identical conditions. The response variable was days alive and the explanatory variable was food level. We extracted the median day of survival at each food level, and associated $\mathrm{p}$-value from a likelihood ratio test of food levels.

\section{D. magna Ageing and CR experiments for WGBS}

Maternal lines of each strain were acclimatised identically to the survival analysis above. Following three generations of acclimatisation, 20 offspring from each mother were isolated to form a biological replicate. We generated six replicates per age and food level for both clones, resulting in 24 replicates per clone and 48 replicates in the experiment in total (summarised in Fig. 1 b). Twelve replicates per clone were fed a normal diet of $5 \times 10^{6}$ algal cells/day and twelve were fed a CR diet of $1 \times 10^{6}$ algal cells/day (as for [3, 59], but differing slightly from the survival analysis). Each replicate was split and reared in four sub-replicate jars of five animals, which were subsequently pooled for DNA extraction. Six replicates of the twelve at each food level and clone were harvested after the first clutch at approximately 10 days (forming 'Young' replicates) and the other six after the fifth clutch at approximately 50 days ('Old' replicates). $D$. magna were ground by motorised pestle in Digsol and proteinase $\mathrm{K}$ and incubated overnight at $37{ }^{\circ} \mathrm{C}$ and stored at $-70{ }^{\circ} \mathrm{C}$ until DNA extraction. DNA was extracted from pooled $D$. magna per replicate by phenol-chloroform followed by a Riboshredder RNA digestion step and repeat of the phenol-chloroform extraction (following [59]). DNA was eluted into $100 \mu \mathrm{l}$ of TE buffer and quantified by Qubit fluorimeter by dsDNA HS Assay Kit. Sample purity was checked by Nanodrop 260:280 and 260:230 and DNA integrity was examined by running approximately $35 \mathrm{ng}$ DNA on a $0.8 \%$ agarose gel stained with ethidium bromide.

\section{Methylated $\mathrm{CpG}$ and variant prediction}

Bisulphite libraries for WGBS were prepared using EpiGnome/TruSeq DNA methylation kits and 150 bp pairedend sequenced on Illumina HiSeq 4000 by the Centre for Genomic Research (Liverpool, United Kingdom), and raw data deposited in the European Nucleotide Archive (PRJEB34509). Reads were provided by CGR post-trimming with Cutadapt v1.2.1 and Sickle v1.200 for adapters and read quality. Cutadapt option-O 3 was used to trim the $3^{\prime}$ end of any reads which matched the adapter sequence for $3 \mathrm{bp}$, and Sickle removed read windows with an average phred-scaled quality score below 20 . Reads shorter than $20 \mathrm{bp}$ after trimming were removed.
Read quality was assessed with FastQC, and the first $15 \mathrm{bp}$ of each read trimmed with fastp v0.19.3 to remove biases introduced by the random-priming library preparation step, and processed reads reassessed with FastQC. We applied the Bismark (v0.22.3) pipeline (with options "-N 1 -score_min L, $0,-0.6$ ") to generate $\mathrm{CPG}$ methylation calls for each replicate using the chromosomal Daphnia magna (KIT strain) genome assembly version as a reference [64]. Bismark mapping rates were compared using Spearman's Rho (in base $\mathrm{R}$ 4.0.0) between this reference and D. magna assembly 2.4, which was the reference used in our previous work [59]. Aligned reads were then deduplicated in Bismark, and we evaluated three filtering methods to reduce the effect of bisulphite reaction non-conversion on methylation calls. Firstly, all reads with methylation at non-CpG sites were removed, this allowed direct comparison with Hearn et al. 2019 and we did this as non-CpG methylation (at $\mathrm{CHH}$ and $\mathrm{CHG}$ positions) is considered negligible in D. magna [55]-referred to as the 'No-CH' dataset throughout. Secondly, we filter reads with three or more consecutive unconverted $\mathrm{CH}$ cytosines as recommended for BS datasets by [63], which we refer to as the ' $3 \mathrm{x} u \mathrm{CH}$ ' dataset here. Both of these methods were implemented through the 'filter non_conversion' tool within Bismark, and the results for unfiltered methylation calls are presented for comparison with the two filtering methods. Finally, CpG read coverages for methylated and unmethylated reads were combined for the forward and reverse strand data per CpG using the Bioconductor package bsseq [68] ( $\mathrm{R}$ code, Additional file 16). Mapping rates and other metrics per replicate were generated in multiQC [87]. To assess inter-strain genetic differences, SNPs were called in BS-SNPer (v1.0) [70] on 'filter_non_conversion' output bam files pooled for each strain (minus sample 5 for C32) with parameters: '-minhetfreq 0.1 -minhomfreq 0.85 -minquali 15 -mincover 10 -maxcover 1000 minread2 2 -errorate 0.02 -mapvalue 20'. SNP calls were filtered at phred-scaled quality 20 and intersected in bcftools (isec, v1.11).

Clustering of replicates was explored by principal components analysis (PCA) in CPGTools [88], and hierarchical clustering using the $\mathrm{R}$ package pvclust [89] of the 10,000 most variable CpG sites ( $R$ code, Additional file 16) with 10,000 bootstrap re-samplings. To do this, methylation status at each position for the ' $3 \mathrm{x} u \mathrm{CH}$ ' dataset was converted into a proportion or beta value and sites with zero methylated cytosines or with no read coverage in a replicate were removed; variances were then ranked per methylated position with beta_topN.py of CPGTools. To explore genome-wide patters of methylation CpG counts were combined 


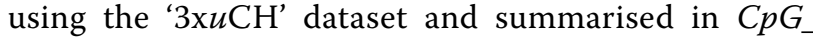
anno_position of CPGTools for CpG sites with greater than 5\% methylation (input gene coordinate BED file: Additional file 18).

\section{Differential methylation testing}

To test the effect of ageing, nutrition, strain and any interactions between them on CpG methylation sites, a generalised linear modelling approach through the Bioconductor package DSS (version 2.36.0 in R version 4.0.0) was developed. Bismark output coverage per replicate was first converted to DSS input format and filtered so that at least two of the six replicates per category (Age $\times$ Food $\times$ Strain) had a coverage of at least two prior to model testing of all factors and their interactions ( Strain + Food + Age + Strain:Food + Strain:Age + Age:F ood + Strain:Food:Age; DSS R script, Additional file 16). CpG sites with an adjusted p-value of less than 0.05 were considered significant. Gene ontology (GO) term enrichment was tested for genes with exons (coding sequence plus untranslated regions) overlapping hypermethylated CpG sites in each strain. Gene ontology annotations were assigned by eggNOG (version 2.0.1) with the predicted protein sequences of the KIT assembly [64] as input. Enrichment was performed in topGO (version 2.40.0) for biological process (BP) and molecular function (MF) categories ( $\mathrm{R}$ code, Additional file 16) for genes with at least one hypermethylated $\mathrm{CpG}$ within exons and the subset of genes with least five hypermethylated CpGs per exonic sequences. The 'weight $01^{\prime}$ algorithm and a p-value threshold of less than 0.01 were applied; $\mathrm{p}$-values were not corrected in line with topGO user manual recommendation (see topGO user manual Sect. 6.2: The adjustment of $\mathrm{p}$-values).

\section{Penalised regression of age significant $\mathrm{CpG}$ sites}

To explore the potential for an epigenetic clock in $D$. magna, we performed a penalised logistic regression on the Age significant CpGs on per replicate beta values implemented using the caret and glmnet $R$ packages [90, 91] (R code, Additional file 16). A lasso (or Least Absolute Shrinkage and Selection Operator) regression was applied to identify coefficients, in this case Age significant CpGs, that contribute most significantly to the model in which Age is the explanatory variable, leaving us with the best $\mathrm{CpG}$ candidates for constructing a clock. The predicted or 'epigenetic age' of each replicate according to these CpGs was extracted from the model and plotted against chronological age (10 or 50 days). Finally, we tested if these epigenetic ages were predicted by Age, Strain and their interaction (Predicted Age $\sim$ Strain + Ag $\mathrm{e}+$ Strain:Age) through a generalised linear model (GLM code, Additional file 16). We extracted effect sizes for Age and Strain by dividing the sum of squares for each factor and their interaction by the total sum of squares. Effect sizes were multiplied by 100 to give percentage of variance explained.

\section{Supplementary Information}

The online version contains supplementary material available at https://doi. org/10.1186/s13072-020-00379-z.

Additional file 1. MultiQC results for the ' $3 \mathrm{xuCH}^{\prime}$ filteressd datasets in htm format.

Additional file 2. MultiQC results for the 'No-CH' filtered datasets in html format.

Additional file 3: Figure S1. PCA plot of replicates for principal components one and two for the top 10000 most variably methylated CpGs by beta value including sample 5 . This sample originates from C32 but clusters with KA53, it represents a mislabelled or contaminated sample and was removed from further analysis; $C 32=$ red, $K A 53=$ green.

Additional file 4: Table S1. Metrics for 5\% plus CpG methylated sites across gene-bodies, generated in (pGTools and used to create Fig. 2c).

Additional file 5: Figure S2. Distribution of CpGs with greater than 5\% methylation combined across gene-bodies and by exon number ( $1=$ closest to transcription start site) separately for each strain, showing that the distributions do not differ between them.

Additional file 6: Table S2. Metrics for 5\% plus CpG methylated sites across exons, generated in CpGTools and used to create Fig. 2d).

Additional file 7: Table S3. Number of DSS significant CpG sites for each factor and their interactions at each filtering level and number of SNPS predicted in each strain by BS-SNPer.

Additional file 8: Table S4. DSS output for significant CpGs for Age and Strain for ' $3 \mathrm{xuCH} \mathrm{CH}^{\prime}$ and ${ }^{\mathrm{N}} \mathrm{No}-\mathrm{CH}^{\prime}$ datasets. Each category occurs consecutively in the file.

Additional file 9: Figure S3. Euler diagram of the overlap between '3xuCH' and 'No-CH' filtering for CpGs significantly differentially methylated between strains

Additional file 10: Table S5. Hypermethylated positions in strain KA53 for gene LOC116934226 by exon.

Additional file 11: Table S6. Enriched GO terms for one or five plus enriched CpGs hypermethylated in each strain for Biological Process and Molecular Function GO categories (3xuCH dataset).

Additional file 12: Table S7. Gene annotations for genes overlapping CpGs significantly associated with ageing in D. magna.

Additional file 13: Table S8. CpGs with a significant contribution to epigenetic age and their coefficients after penalised lasso regression for '3xuCH' and 'No-CH' datasets.

Additional file 14: Table S9. Summary table of GLM results for predicted epigenetic age against Age and Strain ' $3 \mathrm{xuCH}$ ' and 'No-CH' datasets.

Additional file 15: Figure S4. Part A) PCA analysis of beta values for the twelve Age significant $C p G$ s selected by penalised lasso regression. As for Fig. 3a), C32 young individuals cluster separately on P(1. Part B) The relationship between predicted epigenetic age and chronological age for all replicates for the 'No-CH' dataset, coloured by strain and calculated from the $4 \mathrm{CpGs}$ that contributed most to the penalised lasso regression model.

Additional file 16. $R$ code for combining complementary CpGs, Cox's proportional hazards, DSS analysis, topGO analysis, and penalised regression.

Additional file 17. Longevity experiment results for the two strains under caloric restriction and normal food input to the Cox's proportional hazards analyses, which were run separately for each strain. $\mathrm{H}=$ normal food 
individual; $L=$ caloric restricted individual. Individuals were changed to status ' 2 ' on death in line with survival $R$ program requirements.

Additional file 18. Gene component coordinates bed file for the KIT1 $D$. magna genome assembly. Used to calculate $\mathrm{CpG}$ rates per kb for each category shown in Fig. 2c, d.

\section{Abbreviations}

3xuCH: Dataset for which reads with three or more consecutive unconverted $\mathrm{CH}$ cytosines were removed prior to differential methylation testing; bp: Base pairs; BP: Biological process; $\mathrm{CHH/CHG:} \mathrm{Cytosine} \mathrm{followed} \mathrm{by} \mathrm{nucleotides}$ where $\mathrm{H}$ is adenine, thymine or cytosine and $\mathrm{G}$ is guanine; $\mathrm{Cl}$ : Confidence interval; CpG: Cytosine followed by guanine; CR: Caloric restriction; DNA: Deoxyribonucleic acid; DNMT: DNA methyltransferase; DSS: Dispersion shrinkage for sequencing data; ENA: European Nucleotide Archive; FDR: False discovery rate; GLM: Generalised linear model; GO: Gene ontology; lasso: Least Absolute Shrinkage and Selection Operator; MF: Molecular function; MZT: Maternal to Zygotic; NF: Normal food; No-CH: Dataset for which reads with all reads containing $\mathrm{CH}$-methylated cytosines were removed prior to differential methylation testing; PCA: Principal components analysis; PC1/2: Principal component 1 or 2; PCR: Polymerase chain reaction; SNP: Single-nucleotide polymorphism; TE: Tris(hydroxymethyl)aminomethane ethylenediaminetetraacetic acid; UTR: Untranslated region; WGBS: Whole-genome bisulphite sequencing.

\section{Acknowledgements}

Not applicable

\section{Authors' contributions}

TJL and JH designed this study. FP, JH and TJL performed the experiment and DNA extraction. JH analysed the DNA methylation data and interpreted the results, with modelling input from TJL. JH wrote the manuscript with assistance from TJL. All the authors read and approved the final manuscript.

\section{Funding}

This research was funded Institutional Strategic Support Funds (204804/Z/16/Z) awarded to School of Biological Sciences, University of Edinburgh by The Wellcome Trust.

\section{Availability of data and materials}

The sequencing reads generated for this study are available in the European Nucleotide Archive under accession PRJEB34509, https://www.ebi.ac.uk/ena/ browser/view/PRJEB34509 [92]

\section{Ethics approval and consent to participate}

Not applicable.

\section{Consent for publication}

Not applicable.

\section{Competing interests}

Not applicable.

\section{Author details}

1 Department of Vector Biology, Liverpool School of Tropical Medicine, Liverpool, UK. ${ }^{2}$ The James Hutton Institute, Craigiebuckler, Aberdeen, UK. ${ }^{3}$ School of Biological Sciences, University of Aberdeen, Aberdeen, UK. ${ }^{4}$ Institute of Evolutionary Biology, School of Biological Sciences, University of Edinburgh, Edinburgh, UK.

Received: 9 November 2020 Accepted: 12 December 2020

Published online: 06 January 2021

\section{References}

1. Hearn J, Clark J, Wilson PJ, Little TJ. Daphnia magna modifies its gene expression extensively in response to caloric restriction revealing a novel effect on haemoglobin isoform preference. Mol Ecol. 2020. https://doi. org/10.1111/mec.15557.
2. Garbutt JS, Little TJ. Bigger is better: changes in body size explain a maternal effect of food on offspring disease resistance. Ecol Evol. 2017:7:1403-9.

3. Hearn J, Chow FWN, Barton H, Tung M, Wilson P, Blaxter M, et al. Daphnia magna microRNAs respond to nutritional stress and ageing but are not transgenerational. Mol Ecol. 2018;27:1402-12.

4. Xiang H, Zhu J, Chen Q, Dai F, Li X, Li M, et al. Single base-resolution methylome of the silkworm reveals a sparse epigenomic map. Nat Biotechnol. 2010:28:516-20.

5. Hunt BG, Glastad KM, Yi SV, Goodisman MAD. The function of intragenic DNA methylation: insights from insect epigenomes. Integr Comp Biol. 2013:53:319-28.

6. Glastad KM, Arsenault SV, Vertacnik KL, Geib SM, Kay S, Danforth BN, et al. Variation in DNA methylation is not consistently reflected by sociality in Hymenoptera. Genome Biol. 2017;9:1687-98.

7. Glastad KM, Hunt BG, Goodisman MAD. Epigenetics in insects: genome regulation and the generation of phenotypic diversity. Annu Rev Entomol. 2019;64:185-203.

8. Kvist J, Gonçalves Athanàsio C, Shams Solari O, Brown JB, Colbourne JK, Pfrender ME, et al. Pattern of DNA methylation in Daphnia: evolutionary perspective. Genome Biol Evol. 2018;10:1988-2007.

9. Kvist J, Athanàsio CG, Pfrender ME, Brown JB, Colbourne JK, Mirbahai L. A comprehensive epigenomic analysis of phenotypically distinguishable, genetically identical female and male Daphnia pulex. BMC Genomics. 2020:21:17.

10. Foret S, Kucharski R, Pittelkow Y, Lockett GA, Maleszka R. Epigenetic regulation of the honey bee transcriptome: unravelling the nature of methylated genes. BMC Genomics. 2009;10:472.

11. Glastad KM, Gokhale K, Liebig J, Goodisman MAD. The caste-and sexspecific DNA methylome of the termite Zootermopsis nevadensis. Sci Rep. 2016:6:1-14.

12. Bewick AJ, Vogel KJ, Moore AJ, Schmitz RJ. Evolution of DNA methylation across insects. Mol Biol Evol. 2017;34:654-65.

13. Olson CE, Roberts SB. Genome-wide profiling of DNA methylation and gene expression in Crassostrea gigas male gametes. Front Physiol. 2014;5:224.

14. Orozco LD, Rubbi L, Martin LJ, Fang F, Hormozdiari F, Che N, et al. Intergenerational genomic DNA methylation patterns in mouse hybrid strains. Genome Biol. 2014;15:R68.

15. Tryndyak VP, Han T, Fuscoe JC, Ross SA, Beland FA, Pogribny IP. Status of hepatic DNA methylome predetermines and modulates the severity of non-alcoholic fatty liver injury in mice. BMC Genomics. 2016;17:1-12.

16. Xie W, Barr CL, Kim A, Yue F, Lee AY, Eubanks J, et al. Base-resolution analyses of sequence and parent-of-origin dependent DNA methylation in the mouse genome. Cell. 2012;148:816-31.

17. Oey H, Isbel L, Hickey P, Ebaid B, Whitelaw E. Genetic and epigenetic variation among inbred mouse littermates: identification of inter-individual differentially methylated regions. Epigenet Chromatin. 2015;8:54.

18. Ollikainen M, Smith KR, Joo EJH, Ng HK, Andronikos R, Novakovic B, et al. DNA methylation analysis of multiple tissues from newborn twins reveals both genetic and intrauterine components to variation in the human neonatal epigenome. Hum Mol Genet. 2010;19:4176-88.

19. Egger $G$, Liang $G$, Aparicio A, Jones PA. Epigenetics in human disease and prospects for epigenetic therapy. Nature. 2004;429:457.

20. Horvath S. DNA methylation age of human tissues and cell types. Genome Biol. 2013;14:3156.

21. Horvath S. Erratum to: DNA methylation age of human tissues and cell types. Genome Biol. 2015;16:96.

22. Polanowski AM, Robbins J, Chandler D, Jarman SN. Epigenetic estimation of age in humpback whales. Mol Ecol Resour. 2014;14:976-87.

23. Piferrer F, Anastasiadi D, Valdivieso A, Sánchez N, Moraleda J, Ribas L. The model of the conserved epigenetic regulation of sex. Front Genet. 2019;10:857.

24. Wright PGR, Mathews F, Schofield H, Morris C, Burrage J, Smith A, et al. Application of a novel molecular method to age free-living wild Bechstein's bats. Mol Ecol Resour. 2018;18:1374-80.

25. Parrott BB, Bertucci EM. Epigenetic aging clocks in ecology and evolution. Trends Ecol Evol. 2019:34:767-70.

26. Little TJ, O'Toole AN, Rambaut A, Chandra T, Marioni R, Pedersen AB. Methylation-based age estimation in a wild mouse. bioRxiv. 2020. https:// doi.org/10.1101/2020.07.16.203687. 
27. Cardoso-Júnior CAM, Guidugli-Lazzarini KR, Hartfelder K. DNA methylation affects the lifespan of honey bee (Apis mellifera $L$ ) workers-Evidence for a regulatory module that involves vitellogenin expression but is independent of juvenile hormone function. Insect Biochem Mol Biol. 2018:92:21-9.

28. Mathers TC, Mugford ST, Percival-Alwyn L, Chen Y, Kaithakottil G, Swarbreck D, et al. Sex-specific changes in the aphid DNA methylation landscape. Mol Ecol. 2019;28:4228-41.

29. Bain SA, Marshall H, Ross L. Sex-specific expression and DNA methylation in a species with extreme sexual dimorphism and paternal genome elimination. bioRxiv. 2020. https://doi.org/10.1101/2020.06.25.171488.

30. Herb BR, Wolschin F, Hansen KD, Aryee MJ, Langmead B, Irizarry R, et al. Reversible switching between epigenetic states in honeybee behavioral subcastes. Nat Neurosci. 2012;15:1371-3.

31. Claudio-Piedras F, Recio-Tótoro B, Condé R, Hernández-Tablas JM, Hurtado-Sil G, Lanz-Mendoza H. DNA methylation in Anopheles albimanus modulates the midgut immune response against Plasmodium berghei. Front Immunol. 2020;10:3025.

32. Heilbronn LK, Ravussin E. Calorie restriction and aging: review of the literature and implications for studies in humans. Am J Clin Nutr. 2003;78:361-9.

33. Kapahi $P$, Kaeberlein $M$, Hansen M. Dietary restriction and lifespan: Lessons from invertebrate models. Ageing Res Rev. 2017;39:3-14.

34. Lakowski B, Hekimi S. The genetics of caloric restriction in Caenorhabditis elegans. Proc Natl Acad Sci USA. 1998;95:13091-6.

35. Redman LM, Ravussin E. Caloric restriction in humans: impact on physiological, psychological, and behavioral outcomes. Antioxid. 2011;14:275-87.

36. Sohal RS, Weindruch R. Oxidative stress, caloric restriction, and aging. Science. 1996;273:59-63.

37. Walford RL, Harris SB, Weindruch R. Dietary restriction and aging: historical phases, mechanisms and current directions. J Nutr. 1987;117:1650-4.

38. Saiz E, Calbet A, Griffell K, Bersano JGF, Isari S, Solé M, et al. Ageing and caloric restriction in a marine planktonic copepod. Sci Rep. 2015;5:14962.

39. Johnson SC, Rabinovitch PS, Kaeberlein M. mTOR is a key modulator of ageing and age-related disease. Nature. 2013;493:338-45.

40. Hahn O, Grönke S, Stubbs TM, Ficz G, Hendrich O, Krueger F, et al. Dietary restriction protects from age-associated DNA methylation and induces epigenetic reprogramming of lipid metabolism. Genome Biol. 2017;18:56.

41. Kleiven OT, Larsson P, Hobaek A. Sexual reproduction in Daphnia magna requires three stimuli. Oikos. 1992;65:197-206.

42. Innes DJ, Schwartz SS, Hebert PDN. Genotypic diversity and variation in mode of reproduction among populations in the Daphnia pulex group. Heredity (Edinb). 1986;57:345-55.

43. Spitze K, Burnson J, Lynch M. The covariance structure of life-history characters in Daphnia pulex. Evolution (N Y). 1991;45:1081-90.

44. Weider LJ, Hobæk A, Hebert PDN, Crease TJ. Holarctic phylogeography of an asexual species complex-II. Allozymic variation and clonal structure in Arctic Daphnia. Mol Ecol. 1999;8:1-13.

45. Hamrová E, Mergeay J, Petrusek A. Strong differences in the clonal variation of two Daphnia species from mountain lakes affected by overwintering strategy. BMC Evol Biol. 2011;11:231.

46. Sadler DE, Brunner FS, Plaistow SJ. Temperature and clone-dependent effects of microplastics on immunity and life history in Daphnia magna. Environ Pollut. 2019:255:113178.

47. Weber A, Van Noordwijk A. Swimming behaviour of Daphnia clones: differentiation through predator infochemicals. J Plankton Res. 2002;24:1335-48.

48. Weber A, Declerck S. Phenotypic plasticity of Daphnia life history traits in response to predator kairomones: genetic variability and evolutionary potential. Hydrobiologia. 1997;360:89-99.

49. Carvalho GR, Crisp DJ. The clonal ecology of Daphnia magna (Crustacea: Cladocera): I. Temporal changes in the clonal structure of a natural population. J Anim Ecol. 1987:57:453-68.

50. Hietala J, Laurén-Määttä C, Walls M. Life history responses of Daphnia clones to toxic Microcystis at different food levels. J Plankton Res. 1997;19:917-26.

51. De Meester L. Life histories and habitat selection in Daphnia: divergent life histories of D. magna clones differing in phototactic behaviour. Oecologia. 1994;97:333-41
52. Stjernman M, Little TJ. Genetic variation for maternal effects on parasite susceptibility. J Evol Biol. 2011;24:2357-63.

53. Asselman J, De CDIM, Beert E, Janssen CR, Orsini L, Pfrender ME, et al, Bisulfite sequencing with Daphnia highlights a role for epigenetics in regulating stress response to Microcystis through preferential differential methylation of serine and threonine amino acids. Environ Sci Technol. 2017;51:924-31.

54. Asselman J. Bioinformatic analysis of methylation patterns using bisulfite sequencing data. In: Brown SJ, Pfrender ME, editors. Insect genomics methods protoc. New York: Springer; 2019. p. 157-75.

55. Asselman J, De Coninck DIM, Vandegehuchte MB, Jansen M, Decaestecker E, De Meester L, et al. Global cytosine methylation in Daphnia magna depends on genotype, environment, and their interaction. Environ Toxicol Chem. 2015;34:1056-61.

56. Asselman J, De Coninck DIM, Pfrender ME, De Schamphelaere KAC. Gene body methylation patterns in Daphnia are associated with gene family size. Genome Biol Evol. 2016;8:1185-96.

57. Trijau M, Asselman J, Armant O, Adam-Guillermin C, De Schamphelaere KAC, Alonzo F. Transgenerational DNA methylation changes in Daphnia magna exposed to chronic y Irradiation. Environ Sci Technol. 2018;52:4331-9.

58. Jeremias G, Barbosa J, Marques SM, De Schamphelaere KAC, Van Nieuwerburgh F, Deforce D, et al. Transgenerational inheritance of DNA hypomethylation in Daphnia magna in response to salinity stress. Environ Sci Technol. 2018;52:10114-23.

59. Hearn J, Pearson M, Blaxter M, Wilson PJ, Little TJ. Genome-wide methylation is modified by caloric restriction in Daphnia magna. BMC Genomics. 2019;20:197.

60. Lindeman LC, Thaulow J, Song Y, Kamstra JH, Xie L, Asselman J, et al. Epigenetic, transcriptional and phenotypic responses in two generations of Daphnia magna exposed to the DNA methylation inhibitor 5-azacytidine. Environ Epigenet. 2019;5:dvz016.

61. Tsurumi A, Li WX. Aging mechanisms-a perspective mostly from Drosophila. Adv Genet. 2020;1:e10026.

62. Park Y, Wu H. Differential methylation analysis for BS-seq data under general experimental design. Bioinformatics. 2016;32:1446-53.

63. Olova N, Krueger F, Andrews S, Oxley D, Berrens RV, Branco MR, et al. Comparison of whole-genome bisulfite sequencing library preparation strategies identifies sources of biases affecting DNA methylation data. Genome. 2018;19:1-19.

64. Lee B-Y, Choi B-S, Kim M-S, Park JC, Jeong C-B, Han J, et al. The genome of the freshwater water flea Daphnia magna: a potential use for freshwater molecular ecotoxicology. Aquat Toxicol. 2019;210:69-84.

65. Colbourne JK, Pfrender ME, Gilbert D, Thomas WK, Tucker A, Oakley $\mathrm{TH}$, et al. The ecoresponsive genome of Daphnia pulex. Science (80-). 2011:331:555-61.

66. Kowal K, Tkaczyk A, Zkabek T, Pierzchała M, Ślaska B. Comparative analysis of CPG sites and islands distributed in mitochondrial DNA of model organisms. Animals. 2020;10:665.

67. Garbutt JS, Little TJ. Maternal food quantity affects offspring feeding rate in Daphnia magna. Biol Lett. 2014;10:20140356.

68. Hansen KD, Langmead B, Irizarry RA. BSmooth: from whole genome bisulfite sequencing reads to differentially methylated regions. Genome Biol. 2012;13:R83.

69. Lewis SH, Ross L, Bain SA, Pahita E, Smith SA, Cordaux R, et al. Widespread conservation and lineage-specific diversification of genome-wide DNA methylation patterns across arthropods. PLOS Genet. 2020;16:1-24.

70. Gao S, Zou D, Mao L, Liu H, Song P, Chen Y, et al. BS-SNPer: SNP calling in bisulfite-seq data. Bioinformatics. 2015;31:4006-8.

71. Liu Y, Siegmund KD, Laird PW, Berman BP. Bis-SNP: combined DNA methylation and SNP calling for Bisulfite-seq data. Genome Biol. 2012;13:R61.

72. Wang M, Ly M, Lugowski A, Laver JD, Lipshitz HD, Smibert CA, et al. ME31B globally represses maternal mRNAs by two distinct mechanisms during the Drosophila maternal-to-zygotic transition. Elife. 2017;6:e27891.

73. Nakamura A, Amikura R, Hanyu K, Kobayashi S. Me31B silences translation of oocyte-localizing RNAs through the formation of cytoplasmic RNP complex during Drosophila oogenesis. Development. 2001;128:3233-42.

74. Baroux C, Autran D, Gillmor CS, Grimanelli D, Grossniklaus U. The maternal to zygotic transition in animals and plants. Cold Spring Harb. Symp. Quant. Biol. 2008. p. 89-100. 
75. Göertz GP, van Bree JWM, Hiralal A, Fernhout BM, Steffens C, Boeren $\mathrm{S}$, et al. Subgenomic flavivirus RNA binds the mosquito DEAD/H-box helicase ME31B and determines Zika virus transmission by Aedes aegypti. Proc Natl Acad Sci. 2019;116:19136-44.

76. Lewis JJ, van der Burg KRL, Mazo-Vargas A, Reed RD. ChIP-Seq-annotated Heliconius erato genome highlights patterns of cis-regulatory evolution in Lepidoptera. Cell Rep. 2016;16:2855-63.

77. Simola DF, Ye C, Mutti NS, Dolezal K, Bonasio R, Liebig J, et al. A chromatin link to caste identity in the carpenter ant Camponotus floridanus. Genome Res Cold Spring Harbor Lab. 2013;23:486-96.

78. Simola DF, Graham RJ, Brady CM, Enzmann BL, Desplan C, Ray A, et al. Epigenetic (re) programming of caste-specific behavior in the ant Camponotus floridanus. Science (80-). 2016;351:6633.

79. Lim JP, Brunet A. Bridging the transgenerational gap with epigenetic memory. Trends Genet. 2013;29:176-86.

80. Chambeyron S, Seitz H. Insect small non-coding RNA involved in epigenetic regulations. Curr Opin Insect Sci. 2014;1:1-9.

81. Bewick AJ, Sanchez Z, Mckinney EC, Moore AJ, Moore PJ, Schmitz RJ. Dnmt1 is essential for egg production and embryo viability in the large milkweed bug Oncopeltus fasciatus. Epigenetics Chromatin. 2019:12:1-14

82. Ciccarone F, Malavolta M, Calabrese R, Guastafierro T, Bacalini MG, Reale A, et al. Age-dependent expression of DNMT1 and DNMT3B in PBMCs from a large European population enrolled in the MARK-AGE study. Aging Cell. 2016:15:755-65.

83. Clark J, Garbutt JS, McNally L, Little TJ. Disease spread in age structured populations with maternal age effects. Ecol Lett. 2017;20:445-51.

84. Auld SKJR, Hall SR, Housley Ochs J, Sebastian M, Duffy MA. Predator and patterns of within-host growth can mediate both among-host competition and evolution of transmission potential of parasites. Am Nat. 2014;184:S77-90.

85. Klüttgen B, Dülmer U, Engels M, Ratte HT. ADaM, an artificial freshwater for the culture of zooplankton. Water Res. 1994;28:743-6.

86. Therneau TM. survival: A Package for Survival Analysis in R. R package version 3.2-7. R Packag. version 3.2-7; 2020.

87. Ewels $P$, Magnusson M, Lundin S, Käller M. MultiQC: summarize analysis results for multiple tools and samples in a single report. Bioinformatics. 2016;32:3047-8.

88. Wei T, Nie J, Larson NB, Ye Z, Eckel-Passow JE, Robertson KD, et al. CpGtools: a python package for DNA methylation analysis. Bioinformatics. 2019. https://doi.org/10.1093/bioinformatics/btz91.

89. Suzuki R, Shimodaira H. Pvclust: an R package for assessing the uncertainty in hierarchical clustering. Bioinformatics. 2006;22:1540-2.

90. Kuhn $\mathrm{M}$, others. Building predictive models in $\mathrm{R}$ using the caret package.

91. Friedman J, Hastie T, Tibshirani R. Regularization paths for generalized linear models via coordinate descent. J Stat Softw. 2010. https://doi. org/10.18637/jss.v033.i01.

92. Hearn J, Plenderleith F, Little TJ. PRJEB34509: DNA methylation differs widely between strains of the same geographical origin and changes with age in Daphnia magna. European Nucleotide Archive; 2020.

\section{Publisher's Note}

Springer Nature remains neutral with regard to jurisdictional claims in published maps and institutional affiliations.
Ready to submit your research? Choose BMC and benefit from:

- fast, convenient online submission

- thorough peer review by experienced researchers in your field

- rapid publication on acceptance

- support for research data, including large and complex data types

- gold Open Access which fosters wider collaboration and increased citations

- maximum visibility for your research: over 100M website views per year

At BMC, research is always in progress.

Learn more biomedcentral.com/submissions 